\title{
Lab2go - A Repository to Locate Online Laboratories
}

\author{
$\underline{\text { doi:10.3991/ijoe.v6i1.1117 }}$ \\ Christian Maier, Michael Niederstätter \\ Carinthia University of Applied Sciences, Villach, Austria
}

\begin{abstract}
This paper discusses the creation of a common framework to describe online laboratories according to the semantic web technology. The so called Web 3.0 technology is actually growing daily and is proposed to be one of the leading Web technologies within the next years. Improved search mechanisms and facet based browsing are only some key features which enhance the data handling within the repository. Online laboratories are interactive experiments which are available over the Internet and can be divided into two main groups, software simulations and laboratories made up of real hardware equipment. Technology-enhanced learning is becoming a new important trend in higher education worldwide. In particular, engineering education is becoming an exciting emerging field of research because it involves a multitude of disciplines which aim to resolve the pedagogical problems that arise with the advancement of technology. With the help of the semantic web technology it can be made a significant step forward in terms of a general description model for online laboratories and the location of laboratories with requested properties.
\end{abstract}

Index Terms-Online Laboratory, Semantic Web, Ontology, Web Repository

\section{INTRODUCTION}

With the excessive supply of broadband Internet at the end of the last decade many research groups all over the world have started the exploration of ways to supporting and facilitate the learning activities of students by using new possibilities. Important outcomes of these efforts are online laboratories. Since nowadays many modern universities, schools and other organization started programs to offer, in addition to their traditional laboratories, a wide range of online laboratories in different scientific fields. Like traditional laboratories this type of lab provides students with particular engineering experience and allows them to explore systems and their real behaviors. Online laboratories are fundamental for home experimentation because they are especially designed for distant learning students to acquire introductory hands-on experience and familiarize themselves with real-life phenomena. These online experiments can be found in different fields, including electronics, mechatronics, informatics etc. [1].

In traditional laboratories most of the equipment is not efficiently used because of the fact that the labs are used for other experiments or very specific equipment are only used for a very short time period of the year. Online laboratories are a suitable instrument to solve these problems by sharing the labs. Since some years some initiatives were initiated to share labs among different institutes by the use of a common architecture such as the iLab Shared
Architecture from the MIT (Massachusetts Institute of Technology) [2].

Currently, available online laboratories are often hidden from the public education community. The most significant reason for this problem is the current lack of information about online laboratories that provide potentially interested parties the ability to search for adequate laboratories. This lack of information regards almost everyone in the online lab community such as students, administrators as well as professors even if this influence them in a different manner. The fundament of this problem is the lack of information which describes the resources. This is not a specific problem of online laboratories but rather a general problem of the current Web and concerns many content types of special interested communities. A solution to solve this problem can be realized by using Semantic Web technologies to describe these content types, establish a framework and create a base for new searching mechanisms. This paper discusses the creation of an online laboratory portal in the form of a repository, where information about specific properties of these labs is collected. The basic idea behind the Web portal is a semantically linked repository for the elearning community that reduces the efforts of researchers as well as lecturers and students to find and share information about online laboratories all over the world.

\section{ONLINE LABORATORIES}

Online laboratories are interactive experiments that are provided over the Internet. Online labs can be divided into two main groups, software simulations and laboratories made up of real hardware equipment.

Table I describes the classification of laboratories in general. Our focus is the last column of the table.

Software simulations are often used in the field of mathematics and in particular simulations where either the setup of hardware is too expensive or the setup of a laboratory is too difficult or even impossible, due to security reasons. Simulations help students to improve their knowledge and an approximate idea of the behaviour of the "real" world [1].

TABLE I.

CLASSIFICATION OF LABORATORIES

\begin{tabular}{|c|c|c|c|}
\hline \multicolumn{2}{|c|}{} & \multicolumn{2}{c|}{ Experimenter } \\
\cline { 3 - 4 } \multicolumn{2}{|c|}{} & Local & Remote \\
\hline \multirow{3}{*}{ Experiment } & Real & Hand-on-Lab & Remote Lab \\
\cline { 2 - 4 } & Virtual & Local Simulation & Virtual Lab \\
\hline
\end{tabular}


A large amount of currently used software simulations are already Web based and thus allowing learners to get access to these labs at any time and from any place[1] .

Web-based software simulations are so called "Virtual Labs" and differ from Remote Labs in that way, that they only use software while "Remote Laboratories" consist of real hardware equipment. In comparison to "Virtual Laboratories" Remote Laboratories allow persons to manipulate real hardware. Because of the fact that laboratory experiments and instruments are becoming increasingly sophisticated and expensive for universities to purchase and maintain remote laboratories is getting more and more interesting. Remote labs offer a solution and represent a practical alternative through which students may conduct experiments online, regardless of time and space limitations. Compared with traditional laboratory practice, remote labs offer flexible learning in time and place, access to a wide number of distributed experiments and cost-cutting strategies. Furthermore, as collaborative educational strategies become more widespread, remote labs offer great possibilities for students to interact as they work towards a common goal.

\section{SEMANTIC WEB}

At the beginning, the Web consisted basically of many Websites containing only unstructured text. Web 2.0 extended this traditional Web with a few extremely large Web sites specialized on certain specific content types like YouTube for Videos and Flickr for pictures. The transition from Web 2.0 to Web 3.0 implies a growing transition from receiver to producer of information, from static to dynamic contents, from control of the few to the wisdom of the crowds. In contrast to Web 2.0, in Web 3.0 many Web sites will be hosting arbitrarily structured content what will be realised by using Semantic Web technologies.

The fundamental characteristic of the Semantic Web is the description of various content or information with metadata. In the traditional Web anyone can write a page saying whatever they please and publish it to the Web infrastructure. In the case of the Semantic Web, it means that our data infrastructure has to allow the individual to express a piece of data about some entity in a way that it can be combined with information from other sources. This sets some of the foundation for the design of RDF (Resource Description Framework) [3]. It means also that information is not managed for a large corporate data center.

The Semantic Web standards have been created as a medium in which people can collaborate on models that they can use to organize the information and share models that can be used to advance the common collection of knowledge.

The Semantic Web also uses the idea of class hierarchy for representing commonality and variability. Differently to OOP (Object Oriented Programming), Semantic Web is not focused on software representation, classes are not defined in terms of behaviours of methods, although the notation of classes remains and plays much the same role. Higher level classes represent commonality among a large variety of entities and lower level classes represent commonality among a small, specific set of things [4].

This is the essence of modelling in the Semantic Web: providing an infrastructure where not only can anyone say anything about any topic, but an infrastructure that can help a community work through the resulting chaos that is present on the Web concerning different viewpoints about a determined subject [4]. A model can provide a framework (like the described classes and subclasses) for representing and describing commonality and variability of viewpoints when they are known. But, in advance of such an organisation, a model can provide a framework for describing what sorts of things we can say about something.

The Resource Description Framework (RDF) is a W3 Consortium recommendation used today as a general method of modelling information by different syntax formats. The idea of RDF is a metadata model that describes Web resources in the form of subject - predicate object expressions. This form is called triples in the RDF terminology. The subject denotes the resource and the predicate denotes the aspects of the resource and express a relationship between both.

Another important document of the W3 Consortium recommendation is the RDF - Schema (RDFs) which enables the description of so called lightweight ontologies.

\section{ONTOLOGY - BASIC DEFINITIONS}

One of the first steps to make use of the semantic web technology for online laboratories is to create an ontology, or in other words a generalized, formal representation of the domain. This general model consists of various properties, data types and relationships representing various types of online labs in a generic model. To develop a model, which is accepted by the end users it is essential that the model is easy to understand, well structured and a very close representation of the real world situation. At this point several discussions about various general states have to be made to ensure that the model is really applicable to the current real-world-situation.

When starting with the model definition, a lot of questions about very principal things come up. For example how is an online laboratory defined? Should we differ between an experiment and a laboratory? These fundamental questions are very important and only with the right definitions of the basic description model, it can maturate in the right direction.

Because of the various possibilities and terms to describe this domain first of all a general definition of the basic terms and principles in an online lab grid is required.

In our general definition the following terms and definitions are used to describe an online laboratory:

\section{A. Online Laboratory}

An online lab is an environment which allows a person to perform experiments and or simulations over the Internet. Online laboratories consist basically of software based simulations or hardware based experiments.

An online laboratory can be divided further in the following three types of labs:

\section{B. Remote Laboratory}

A remote lab is an online lab which provides real experiments. This definition implies the control of real hardware and the realization of real measurements. 


\section{Virtual Laboratory}

A virtual lab is an online lab which provides software simulations or applications.

\section{Hybrid Laboratory}

A hybrid lab is an online lab which combines virtual lab and remote lab technologies. It provides real hardware experiments and software simulations as well. All types of online labs have several properties, which are described more in detail in Table II.

TABLE II.

PROPERTIES OF AN ONLINE LAB

\begin{tabular}{|c|c|}
\hline Property & Description \\
\hline Access URL & $\begin{array}{l}\text { Every online lab has a URL (Unique Recourse } \\
\text { Locator) which gives access to the online lab } \\
\text { directly or via a middleware. In the last case, } \\
\text { different labs can have the same access URL if } \\
\text { they are connected to the same middleware and } \\
\text { one lab can have one or more access URL if it is } \\
\text { connected to different middleware. }\end{array}$ \\
\hline URI & $\begin{array}{l}\text { The property URI is the Unique Identifier of the } \\
\text { Online Lab. }\end{array}$ \\
\hline Name & $\begin{array}{l}\text { The property title describes the name of an online } \\
\text { lab which has not to be unique. (An online lab can } \\
\text { represent in different languages). }\end{array}$ \\
\hline Experiments & $\begin{array}{l}\text { The property experiments represent virtual and } \\
\text { real experiments of online labs. }\end{array}$ \\
\hline Owner & $\begin{array}{l}\text { The property owner represents a person or an } \\
\text { organization (like universities or companies) } \\
\text { which offers the online lab. }\end{array}$ \\
\hline Administrator & $\begin{array}{l}\text { The property Administrator represents a person } \\
\text { which is responsible for the online lab. }\end{array}$ \\
\hline Creator & $\begin{array}{l}\text { The property Creator represents a person which is } \\
\text { the developer of the online lab }\end{array}$ \\
\hline Description & $\begin{array}{l}\text { The property description is a textual description } \\
\text { about the online laboratory without semantically } \\
\text { linked data which is available in different lan- } \\
\text { guages. }\end{array}$ \\
\hline Languages & $\begin{array}{l}\text { The property language represents the available } \\
\text { languages of the online lab. }\end{array}$ \\
\hline Release date & $\begin{array}{l}\text { The property release date represents the release } \\
\text { date of the laboratory }\end{array}$ \\
\hline $\begin{array}{c}\text { Access } \\
\text { Requirements }\end{array}$ & $\begin{array}{l}\text { The property access requirements will give infor- } \\
\text { mation about the access of the online lab (open } \\
\text { access, access upon request, restricted access) }\end{array}$ \\
\hline Lab Status & Defines whether the laboratory is online or offline. \\
\hline Costs & This property represents the access costs \\
\hline Technical Data & $\begin{array}{l}\text { This property provides information about the } \\
\text { technical background for the online laboratory } \\
\text { developers }\end{array}$ \\
\hline $\begin{array}{l}\text { Client Require- } \\
\text { ments }\end{array}$ & $\begin{array}{l}\text { This property provides information about require- } \\
\text { ments for the clients. (Client Technology, Runtime } \\
\text { engine, Client OS, Browser) }\end{array}$ \\
\hline Documentation & $\begin{array}{l}\text { Documentation of the online Lab including Hard- } \\
\text { ware and Software }\end{array}$ \\
\hline Architecture & $\begin{array}{l}\text { This property describes the architecture that a } \\
\text { specific lab belongs to. }\end{array}$ \\
\hline
\end{tabular}

As already mentioned an online lab can have one or more experiments. An experiment is defined as follows:

\section{E. Experiment}

An experiment in our domain is defined as the smallest enclosed unit of an online laboratory. It provides the execution of virtual or real experiments to observe the behavior and output of a system. An online laboratory consists of one or more experiments in different fields of science and engineering.

Moreover according to the interactivity between experiment and experimenter an experiment can be categorized further:

\section{1) Observation Experiment}

The experiment parameters as well as the experiment environment are fixed. This kind of experiments allows users only the observation of an experiment.

\section{2) Fixed Experiment}

The experiment environment is fixed but the experiment parameters are tunable. Furthermore it is possible to control one or more measurement instruments remotely.

\section{3) Adaptive Experiment}

The experiment parameters as well as the experiment environment are changeable. This definition includes for example the modification of a circuit.

Every experiment of an online laboratory is described by certain properties which are defined in Table III.

TABLE III.

PROPERTIES OF AN EXPERIMENT

\begin{tabular}{|c|c|}
\hline Property & Description \\
\hline URI & $\begin{array}{l}\text { The property URI is the Unique Identifier of the } \\
\text { Online Lab. }\end{array}$ \\
\hline Name & $\begin{array}{l}\text { The property title describes the name of an online } \\
\text { lab which has not to be unique. (An online lab can } \\
\text { represent in different languages). }\end{array}$ \\
\hline $\begin{array}{l}\text { Type of Experi- } \\
\text { ment }\end{array}$ & $\begin{array}{l}\text { The property type of experiment describes the type } \\
\text { of the Experiment (Virtual experiment or Real } \\
\text { experiment) }\end{array}$ \\
\hline Description & $\begin{array}{l}\text { The property description is a textual description } \\
\text { about the experiment without semantically linked } \\
\text { data which is available in different languages. }\end{array}$ \\
\hline Scientific Field & $\begin{array}{l}\text { The property field represents the division of the } \\
\text { experiment like engineering, science as well as } \\
\text { their subdivisions like electronic, mechatronics ... }\end{array}$ \\
\hline Educational level & $\begin{array}{l}\text { The property educational level represents the } \\
\text { current educational level (primary, secondary, } \\
\text { tertia, research) }\end{array}$ \\
\hline Creator & $\begin{array}{l}\text { This property represents a person which is the } \\
\text { creator of the experiment. }\end{array}$ \\
\hline $\begin{array}{l}\text { E-learning mate- } \\
\text { rial }\end{array}$ & $\begin{array}{l}\text { This property informs about additional e-learning } \\
\text { material for the experiment }\end{array}$ \\
\hline Difficulty level & $\begin{array}{l}\text { This property represents the level of difficulty of } \\
\text { the experiment. }\end{array}$ \\
\hline Online lab & $\begin{array}{l}\text { An experiment is part of one online lab. This } \\
\text { property represents these online labs. }\end{array}$ \\
\hline Documentation & $\begin{array}{l}\text { Documentation of the experiment including Hard- } \\
\text { ware and Software }\end{array}$ \\
\hline Duration & $\begin{array}{l}\text { The time a user needs to complete the remote } \\
\text { experiment. }\end{array}$ \\
\hline
\end{tabular}




\section{TERMINOLOGY}

For the terminology existing vocabularies and bindings were inspected more in detail. The focus was on several well known standards such as Dublin Core [5], LOM [6], SKOS [7], vCard [8], FOAF [9] and WGS84 [10]. With the analysis of the various standards it could be seen that mostly only parts of the standards can be adopted and that a definition of various new terms for the definition of an online laboratory model is required.

After the evaluation of the different standards it was made the decision to adopt basic terminology and data types from Dublin Core to the model. Examples for adopted terms are such as title, description or the type date which is also defined in Dublin Core.

A further intention is not to make use of SKOS and LOM in the online laboratory description. This is because only small parts are fitting to our problem and can be used to describe the online laboratory model.

Anyway it was decided to adopt some basic terminologies from the not included standards. An example here is the property difficulty from LOM [6]. This property describes very well the difficulty level with a pre-defined range [Very Easy, Easy, Medium, Difficult, Very Difficult].

Besides describing all technical aspects of the Lab itself, a model to describe persons, organizations and projects has to be found. This will be used for properties like creator, administrator or rights holder. As already mentioned vCard and FOAF were inspected more in detail. The decision was to make use of the FOAF ontology because on one hand it allows more possibilities to define relationships between agents and on the other it is much more used as vCard in RDF representation. Within the portal it would be very useful to see which person knows whom. It facilitates then the organization and establishment of connections with other persons and groups of researchers more easily.

\section{ONTOLOGY DESIGN}

The first milestone is to develop an ontology which is easy to understand and to apply. Right now for the first approach the ontology includes only fundamental descriptions and properties which are not too detailed. It is advisable not to implement a too detailed description at the beginning, so that the users have some freedom and the model can grow with additional user's feedback regarding inputs and terminologies. With this practice a creation of an ontology which can't be understood and would not be accepted by the end-user could be avoided. It is a very important point in defining a description model for a domain, as it could help users to get in touch with the description model and gives them also the possibility to contribute to the model development, what might increase the acceptance of the model by the users.

Based on the basic definitions which are described in section $\mathrm{V}$ and on the use of standard vocabulary described in section VI the first draft of the ontology was made. Before starting to build the ontology a class diagram for the model was designed.

As can be seen in figure 1 we used two main classes to describe an Online Laboratory and an Experiment.

The online laboratory class contains all properties which were defined in table IV. The different types of laboratories are designed as subclasses of the online laboratory class. Depending on the type of experiment each subclass is linked to one or more experiment of a specific type. It is obvious that a class of type remote laboratory can be linked only to experiments where the property typeOfExperiment is set to "Real experiment". Similar it is for the classes virtual and hybrid laboratory.

The Experiment class contains all properties which are described in table IV. Additionally three subclasses were created to determine whether the experiment is an observation, a controlled or an adaptive experiment. With this further division it is easier for users to differ between the

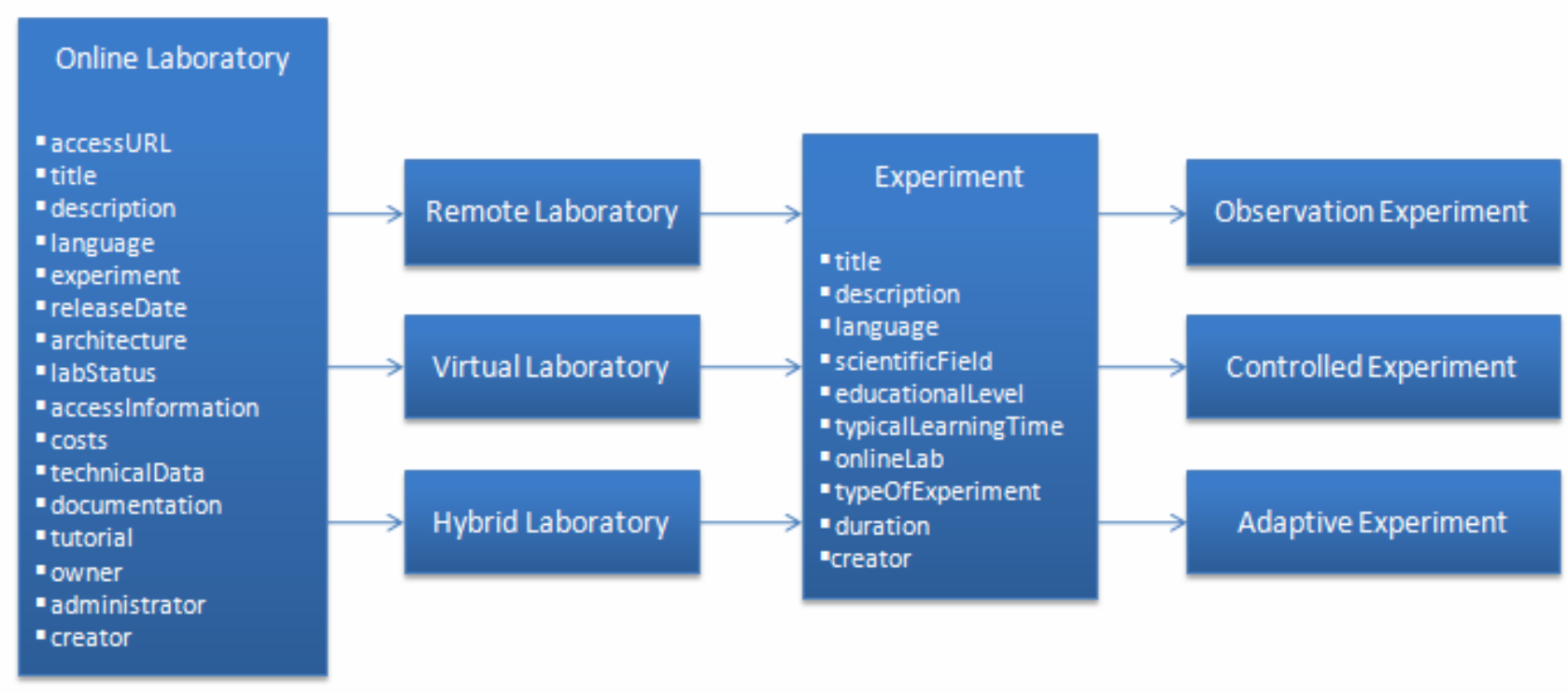

Figure 1. The class structure of the ontology 
interactivity types of experiments. To differ trough various interactivity types also a further property in the class Experiment could be used, but in terms of model readability and the implementation of facet based browsing it is much better to make use of subclasses.

Afterwards the Software Protégé [9] was used to design the ontology itself. Protégé is a freely available tool to design ontologies. It has a lot of useful plug-ins and extensions such as the reasoner Pellet to check the ontology's consistence and the property Matrix plug-in make the ontology design easier. That plug-ins will help through the development process.

As already mentioned above, the plan is to let the ontology grow together with the user inputs. It is now questionable how this can be realized? We assume that users would not send e-mails or insert various comments in a forum if they have any improvements. Therefore next to the definition of the properties in our ontology the user has the possibility to add additional information. This will be realized similar as in Wikipedia. The user could write additional descriptions by setting tags. The evaluation and analysis of these tags will give the developer very useful hints to find out which additional properties are desired by the users. Afterwards it is apparently which tags can be adapted to the model as a new property or also which properties can be renamed that they are used in the proposed way.

\section{THE INTENTION OF LAB2GO}

The basic idea of the Web portal is a repository that offers a common framework to collect and describe laboratory data from different laboratory providers located all over the world turns out to be necessary to continually foster the development of laboratories and exchange of knowledge among interested parties. This Web Portal will host information about running research projects, researchers, organisations, existing state of the art technologies, etc, in order to strengthen the collaboration in that field of science as well as knowledge about the laboratories and their operating institutes.

To solve this problem this paper shows the fundamental problems that exist today and the resulting requirement which are necessary to implement a successful platform. One intention for the platform is the improvement of the search process for online laboratories with the use of Semantic Web technologies. Due to the particular way used by the Semantic Web to describe resources, not only full-text search can be supported. This kind of description enables new ways for the implementation of search mechanisms like faced based browsing which allows the user to search information according to the properties of a special object. Furthermore it is possible to query resources based on specific criteria. To outline the differences between the well known Web 2.0 methods and the Semantic Web a typical information searching scenario can be used:

- A user search for all online labs which contains experiments in a specific filed, has a specific difficulty level and is freely available.

Using today's available Web searching tools such a query is not possible as these tools make use of keywords to perform a search. On the other hand such a scenario is perfectly supported by the Semantic Web. This new possibilities provided by the Semantic Web is one of the important keys to advance the information exchange in the community.

The fundamental concept of this project is based on the idea of making use of already existing solutions. Therefore the semantic collaboration platform OntoWiki which was developed by the research group AKSW at InFAI (Universität Leipzig, Institut für Angewandte Informatik) was chosen. OntoWiki is an open-source Platform which can be installed by any Web space and accessed by an ordinary Web browser [11] and is easily adjustable by writing customized plug-ins. Differently to MediaWiki [12], the base platform of Wikipedia, where the metadata can be directly included into the text, OntoWiki is completely based on tags and can be called as a data wiki.

OntoWiki is the base for the portal and provides a framework for the development of Semantic Web application and was therefore used to create a customized solution for online laboratories. Mostly users are not familiar with the concepts of Semantic Web or are not willing to spend time writing the metadata manually. For that reasons Lab2go is also a tool to create metadata for the online laboratory resources.

\section{The Functionalities OF THE PORTAL}

The focus of this chapter is to point out the main functionalities which will be available within the lab2go repository.

canal users have the right to browse through the repository content and search information about online laboratories. With a free registration a user gets the rights to add, edit and evaluate content. In terms of evaluation there are the possibilities to rate and to comment a resource. With this functionality a user gets a better feeling of the relevance of a resource.

A significant new functionality is the searching possibility. With the help of facet based browsing customized views can be generated. Property values can be filtered out from a list of resources. This so called filtering can be applied to any type of resource and also to any relation within the description model. With a combination of two or more filters the list of results becomes mode precise. Another possibility is to display various properties directly in a resource list that the property values can be recognized very quickly.

To manage the data tools like an editor were developed. It looks like a common html-form when entering new lab data or changing some contents. In the backend the data is converted automatically to metadata and then saved in the store. Features like auto complete for predefined property values or resource names (if already existing) make the data input more comfortable. Also visual inputs like a calendar or the possibility to add multiple values of a property makes the editing of resources easier.

Additionally to the pre-defined description model a user can set individually tags for each resource. This has the advantage that in case a user wants to describe his lab with a specific term or property he has the freedom to do this without any limitation. In a second stage exactly these tags can be inspected and evaluated. The result represents a significant input for the ontology development. Misuse of terminology and need for additional terminology could be detected easily. 
Not only searching, evaluation and data management tools were implemented in lab2go. One of the most important key points remains as in so many applications the data handling. Filter out data which is not interesting for certain views; finding the right order of the displayed information and make use of an impressive style are only a few considered key points.

\section{CONCLUSION}

Semantic Web technology is a very broad field that can be applied in many distinct areas. In this paper a very specific use-case scenarios are mentioned. There exists however many other possible extensions beyond the main scope described here. It proposes an approach on how to provide such specific type of information using Semantic Web technologies, and comprehends a first attempt of implementation of an open source platform.

Summarizing this paper shows an overview about the Semantic Web and the most important differences to Web 2.0. Furthermore it covers the problems of lack of information channels for online labs and presents a possible solution in the form of an online portal.

\section{REFERENCES}

[1] M. E. Auer, A. Pester and D.G Zutin, Open Source Portal for Online Laboratories, 2007.

[2] http://icampus.mit.edu/ilabs/architecture, last visited 25.05.2009
[3] Resource Description Framework. URL: http://www.w3.org/RDF/ - Last visited in 03/2009.

[4] D. Allemang ,J. Hendler, Semantic Web for the Working Ontologist: Effective Modeling in RDFS and OWL, Elsevier, 2008.

[5] Dublin Core. URL: http://dublincore.org/

[6] Draft Standard for Learning Object Metadata, IEEE-publication 2002 - URL: http://ltsc.ieee.org/wg12/

[7] SKOS - Simple knowledge Organization System. URL: http://www.w3.org/2004/02/skos/

[8] vCard. URL: http://www.w3.org/TR/vcard-rdf

[9] Protégé - an ontology editor and knowledge-base framework URL: http://protege.stanford.edu/

[10] World Geodetic System. URL: http://www.w3.org/2003/01/geo/

[11] http://www.ontowiki.net, last visited 03.04.2009

[12] http://www.mediawiki.org/wiki/MediaWiki, last visited 25.05.2009

\section{AuTHORS}

C. Maier is with System Engineering Department, Carinthia University of Applied Sciences, Villach, Austria (e-mail: c.maier@fh-kaernten.at).

M. Niderstätter is with System Engineering Department, Carinthia University of Applied Sciences, Villach, Austria (e-mail: m.niederstaetter@fh-kaernten.at).

This work was supported by the European Union founded OntoWiki (FP7-SME-2007-1) project.

Submitted, October, 20, 2009. Published as resubmitted by the authors on January, 20, 2010. 